\title{
Outcome improvement in cerebral ventriculitis after ventricular irrigation: a prospective controlled study
}

\author{
Ahmed Al Menabbawy, MBBCh, MSc, ${ }^{1,2}$ Ehab El Refaee, MD, MSc, ${ }^{1,2}$ \\ Mohamed A. R. Soliman, MD, MSc, ${ }^{1}$ Mohamed A. Elborady, MD, MSc, ${ }^{1}$ \\ Mohamed A. Katri, MBBCh, ${ }^{1}$ Steffen Fleck, MD, PhD, ${ }^{2}$ \\ Henry W. S. Schroeder, MD, PhD, ${ }^{2}$ and Ahmed Zohdi, MD, MSc ${ }^{1}$ \\ 1Department of Neurosurgery, Cairo University, Cairo, Egypt; and 'Department of Neurosurgery, University Medicine Greifswald, \\ Germany
}

OBJECTIVE Cerebral ventriculitis remains one of the most challenging neurosurgical conditions, with poor outcome and a long course of treatment and duration of hospital stay. Despite the current conventional management plans, i.e., using antibiotics in addition to CSF drainage, the outcome remains unsatisfactory in some cases, with no definitive therapeutic guidelines. This study aims to compare the outcome of ventricular irrigation/lavage (endoscopic irrigation or the double-drain technique) to conventional currently accepted therapy using just drainage and antibiotics.

METHODS The authors conducted a prospective controlled study in 33 patients with cerebral ventriculitis in which most of the cases were complications of CSF shunt operations. Patients were divided into two groups. Removal of the ventricular catheter whenever present was performed in both groups. The first group was managed by ventricular lavage/ irrigation, while the other group was managed using conventional therapy by inserting an external ventricular drain. Both systemic and intraventricular antibiotics were used in both groups. The outcomes were compared regarding mortality rate, modified Rankin Scale (mRS) score, and duration of hospital stay.

RESULTS The mean age of the study population was $5.98 \pm 7.02$ years. The mean follow-up duration was $7.6 \pm 3.2$ months in the conventional group and $5.7 \pm 3.4$ months in the lavage group. The mortality rate was $25 \%(4 / 16)$ in the lavage group and $52.9 \%(9 / 17)$ in the nonlavage group $(p=0.1)$. The $\mathrm{mRS}$ score was less than 3 (good outcome) in $68.8 \%$ $(11 / 16)$ of the lavage group cases and in $23.5 \%(4 / 17)$ of the conventional group $(p<0.05)$. The mean hospital stay duration was $20.5 \pm 14.2$ days in the lavage group, whereas it was $39.7 \pm 16.9$ days in the conventional group $(p<0.05)$.

CONCLUSIONS Ventricular lavage or irrigation together with antibiotics is useful in the management of cerebral ventriculitis and associated with a better outcome and shorter hospital stay duration compared to current conventional lines of treatment.

https://thejns.org/doi/abs/10.3171/2020.5.PEDS2063

KEYWORDS cerebral ventriculitis; endoscopic irrigation; ventricular lavage; cerebrospinal fluid infections

$\mathrm{M}$ ORTALITY rates range from $30 \%$ up to $71 \%$ in cases of cerebral ventriculitis despite current therapeutic management plans. ${ }^{1-3}$ The high rates of mortality are usually attributed to multidrug-resistant and gram-negative organisms. Concerning the outcome, only a small portion of surviving individuals can lead a normal life and live independently. Usually, neurosurgical CSF infections are associated with intraventricular catheters (external ventricular drain [EVD] or ventriculoperitoneal [VP] shunts), which adds to the difficulty of treating such infections under current treatment regimens. ${ }^{1,3,4}$ Moreover, they can complicate other neurosurgical procedures, including endoscopic transnasal surgeries. ${ }^{1,5,6}$

Few studies have been published on evaluating the effectiveness of ventricular irrigation or lavage in treating cerebral ventriculitis., ${ }^{3,-12}$ Most of these studies are case reports or case series. Some retrospective comparative studies are also available comparing irrigation/lavage to normal conventional methods of treatment using ventricular catheters and intraventricular and systemic antibiot- 


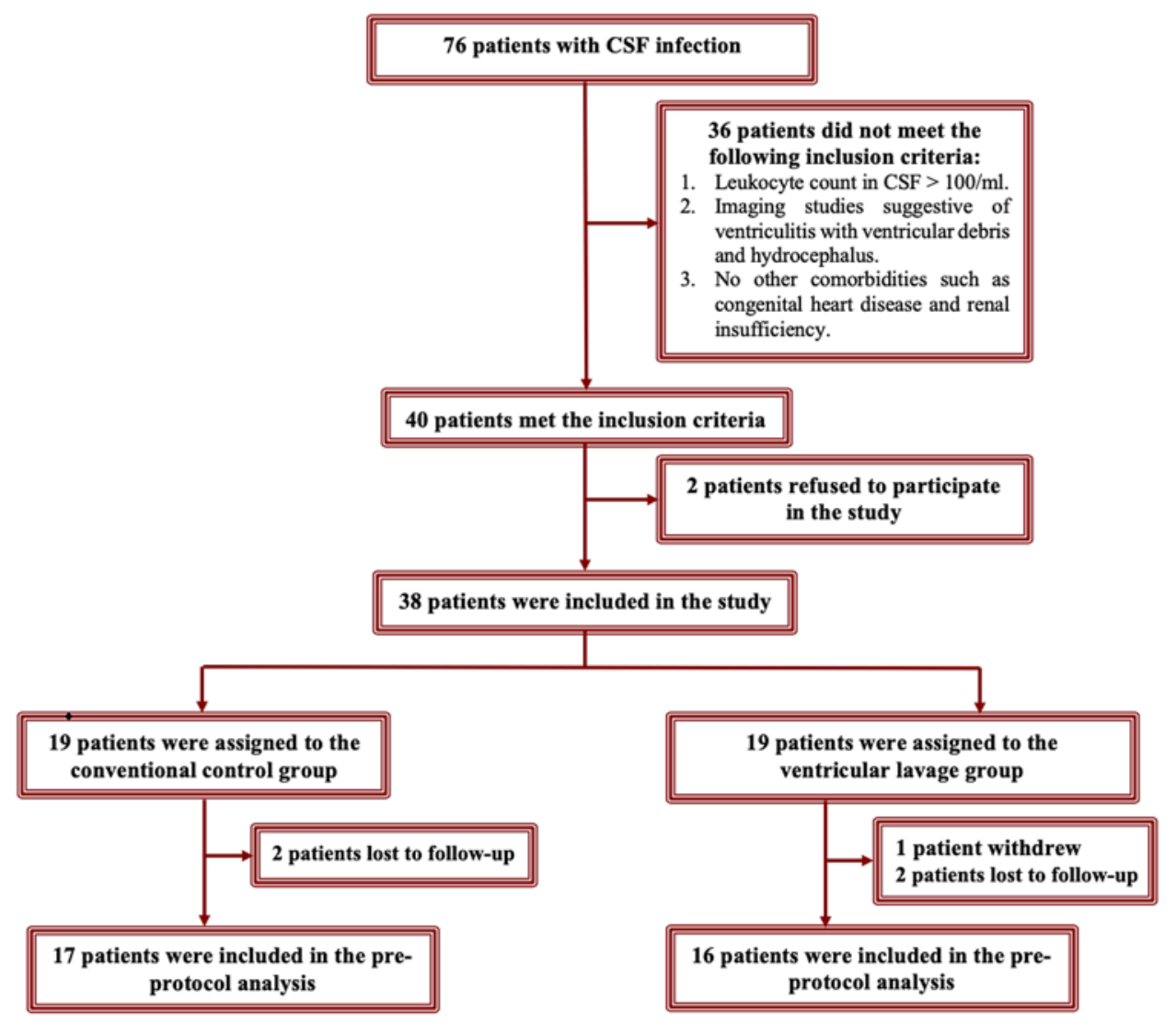

FIG. 1. Flowchart illustrating patient selecting criteria until we reached our target sample. Figure is available in color online only.

ics. ${ }^{12}$ One prospective study was conducted that focused on gram-negative organisms. ${ }^{7}$ However, to our knowledge, comparative prospective studies are currently still lacking. We planned a prospective comparative study comparing ventricular lavage to conventional treatment methods in cerebral ventriculitis.

\section{Methods}

This was a single-center, prospective study conducted in accordance with the ethical committee conditions of Cairo University. All information was collected prospectively and with the willingness of the patients and their parents.

\section{Patient Selection}

To determine the sample size, we used ClinCalc online software with the following parameters: choosing two independent groups (control group and lavage group); dichotomous; power of $80 \%$; alpha level of 0.05 ; enrollment ratio of 1 ; and using the incidence of the primary outcome (mortality) in a similar study from Terada et al., who reported a mortality rate of $37.5 \%$ in the control group and $0 \%$ in the irrigation/lavage group. ${ }^{12}$ The result was a minimum of 32 subjects with at least 16 subjects in each group.

Over a period of 2 years, 76 patients with CSF infections presented to our pediatric neurosurgery department. Only 40 of these patients met our inclusion criteria: leuko- cyte count in CSF more than 100/ml, imaging studies suggestive of ventriculitis as ventricular debris, and septations and/or multilocular hydrocephalus (e.g., MRI; high-signalintensity, T1/T2-weighted, diffusion-weighted imaging; or ultrasonography). We excluded patients with poor general conditions and other comorbidities, such as children with congenital heart disease and renal insufficiency. Two patients refused to participate in the study. One patient chose to discontinue the study and leave the hospital, and with the parents we arranged a transfer to another hospital. Four patients were lost to follow-up. Ultimately, 16 patients in the lavage group and 17 patients in the control group were included in the preprotocol analysis (Fig. 1).

\section{Management}

In all patients, the VP shunt hardware was removed whenever present. In the conventional therapy group (control group), an EVD was inserted, and treatment followed with intraventricular and systemic antibiotics according to the results of the microbiological culture and sensitivity test of the CSF samples. The empirical usage of antibiotics was determined after consulting our clinical pharmacist and pediatrician. CSF samples were withdrawn regularly (every 3 days) until they showed no growth and cell counts of fewer than 30 cells (at least for 2 successive times) together with a decrease in the inflammatory markers and absence of fever. This was followed by removal of the EVD within 1 week, and a VP shunt was placed via a 

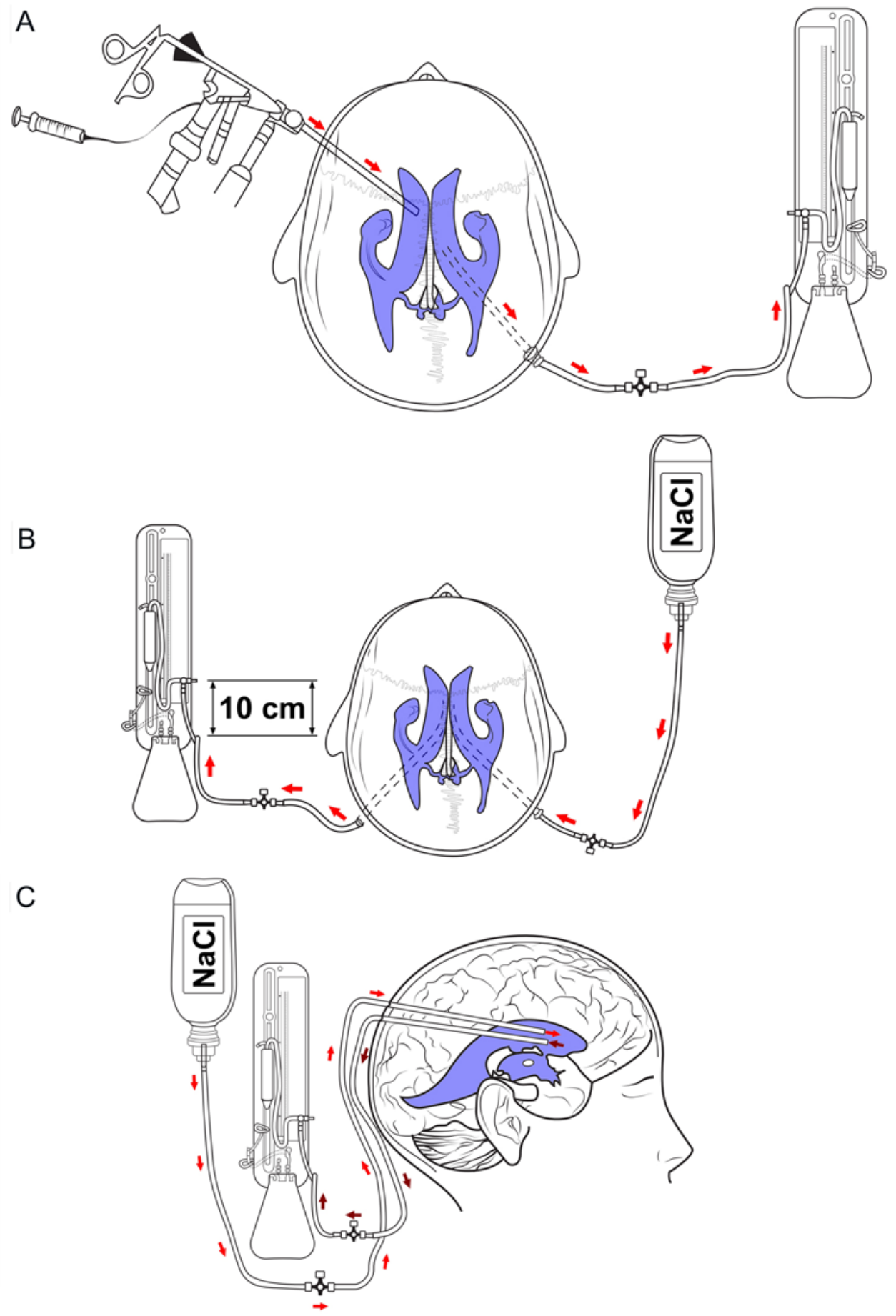

FIG. 2. Illustration of the intraventricular irrigation/lavage techniques including endoscopic lavage (A) and the double-tube technique (B and C). The ventricles with CSF are represented in purple. Copyright Ehab El Refaee. Published with permission. Figure is available in color online only.

new burr hole. This strategy applied to both groups after resolution of the infection.

In the lavage group, ventricular irrigation was performed either by using the irrigation channel of the endoscope (Fig. 2A) or by using a "double-tube technique" without using the endoscope, as shown in Fig. 2. In both techniques, 2 burr holes were used, one for irrigation and the other for drainage, except for 1 patient in whom endoscopic irrigation and drainage were performed via the same burr hole after endoscopic visualization and exclusion of visible ventricular system obstruction. Radiological evidence of septations or univentricular hydrocephalus 
denoting foramen of Monro obstruction was a determining factor in choosing the endoscopic lavage technique rather than the double-tube technique. Also, in the endoscopic irrigation group, an irrigation catheter was inserted at the end of the operation to apply future irrigation on the ward, except for 1 patient who had only 1 burr hole. This patient did not need further irrigation because the leukocytic count in CSF fell below 100 after the first and only irrigation.

The double-tube technique is illustrated in Fig. 2B and C. It shows that the usual drainage system was inserted on one side while another ventricular catheter was inserted on the other side. The level of the draining catheter was always kept at $10 \mathrm{~cm}$ above the tragus of the ear to avoid lowering intracranial pressure and the development of pneumocephalus. In the endoscopic lavage patients, visualization of the dependent posterior horn of the lateral ventricle was performed by planning a precoronal burr hole and debris was washed out from lateral ventricles under vision; where necessary, a septostomy was also performed.

Intraoperative lavage was performed using $1 \mathrm{~L}$ of normal saline $(\mathrm{NaCl} 0.9 \%)$ and $500 \mathrm{mg}$ of vancomycin over 30 minutes. Postoperatively in the operating room, the 2 catheters were kept in place so that the draining catheter worked as an EVD while the irrigating catheter was closed and sealed with tape. In the ward, the treatment followed using intraventricular and systemic antibiotics according to the results of the microbiological culture and sensitivity test with the same amount of saline and vancomycin, but over 4 hours. Three days later, a new CSF sample was taken 12 hours after the last intraventricular antibiotic injection, to avoid diluted CSF and falsified results. If the leukocyte count in the new CSF sample was more than 100 cells $/ \mathrm{ml}$, irrigation was repeated once again in the ward, and a new sample was obtained after 3 days. If the leukocyte count was less than 100 cells $/ \mathrm{ml}$, the irrigation catheter was removed and therapy continued only with antibiotics and drainage. The same criteria for removing the EVD mentioned in the control group were also applied to the lavage group (Fig. 3).

The intraventricular antibiotic of choice was vancomycin, and whenever the causative organisms proved resistant to vancomycin, intraventricular antibiotics were optimized according to the results of the culture and sensitivity test. Other intraventricularly used antibiotics included amikacin and polymyxin E (colistin).

Systemic antibiotics were also tailored according to the culture and sensitivity test results. In many cases, due to the severity of the infections and multiresistance of the organisms, it was obligatory to use antibiotics with a relatively high spectrum of possible side effects. The decision, dosing, and close clinical and biochemical laboratory follow-up regarding possible side effects were always communicated with our pediatricians and clinical pharmacist.

The position of the burr holes depended largely on the site and size of ventricular dilation. We performed 2 frontal burr holes, 2 parietal burr holes, and 1 parietal and 1 frontal burr hole. It is of crucial importance to leave one clean area for a future burr hole for the VP shunt, after the resolution of infection.

To minimize the possible human variation in perform- ing the operative technique, all cases were operated on by a neurosurgical team of 1 consultant (E.E.R.) and 1 resident (A.A.M.), who are the first 2 authors of this study.

\section{Postoperative Assessment}

Patients were closely monitored during their hospital stay, and depending on their clinical state, they were admitted to either the ward (whenever possible to avoid infections from the intensive care unit) or intermediate or intensive care units. Deaths and hospital stay duration were documented. Morbidity was assessed in the outpatient clinic follow-up visits using the modified Rankin Scale (mRS).

\section{Statistical Analysis}

The unpaired Fisher exact test, Student t-test, and chisquare test were used as appropriate to analyze differences between both groups in the demographics, including age, sex, follow-up period, preoperative presentation symptoms (fever, disturbed consciousness level, vomiting, convulsions, abdominal pain, and meningeal irritation), average hospital stay, and postoperative clinical outcome (mortality rate and mRS score, including gram-positive and gram-negative organisms). Statistical significance was established at $\mathrm{p}<0.05$.

\section{Results \\ Demographics}

The demographic data, mean follow-up period, and clinical presentations of each group are presented in Table 1. Detailed data of the predisposing factors, causative organisms, tailored systemic antibiotics, and mRS scores in each case are presented in Tables 2 and 3 for the conventional and irrigation groups, respectively. Demographics did not vary significantly between the two groups, nor did the follow-up period. The study was conducted on the pediatric age group with an average age of $5.98 \pm 7.02$ years, including 3 neonates younger than 28 days old; two were included in the lavage group and the other was included in the conventional group, with no perioperative complications related to the technique. The average age of patients included in each group is shown in Table 1, without a significant difference between the two groups.

The primary and secondary outcomes are presented in Table 4 . The lavage group showed statistically significant differences in outcome regarding mRS scores and hospitalization periods. The worst outcomes in patients according to the causative organisms were gram-negative bacterial and fungal infections, but this was not statistically significant ( $\mathrm{p}=0.13$ and 0.22 respectively). In addition, there was a significantly better outcome in the irrigation/ lavage group compared to the conventional group for patients infected with gram-negative bacteria $(\mathrm{p}<0.05)$ and a nonsignificantly better outcome in patients infected with gram-positive bacteria $(\mathrm{p}=0.058)$ and in patients who failed to grow bacteria $(\mathrm{p}=0.41)$.

\section{Complications}

Complications occurred in 8/33 patients (24.2\%). Shunt obstruction after placement of the new shunt was the most 


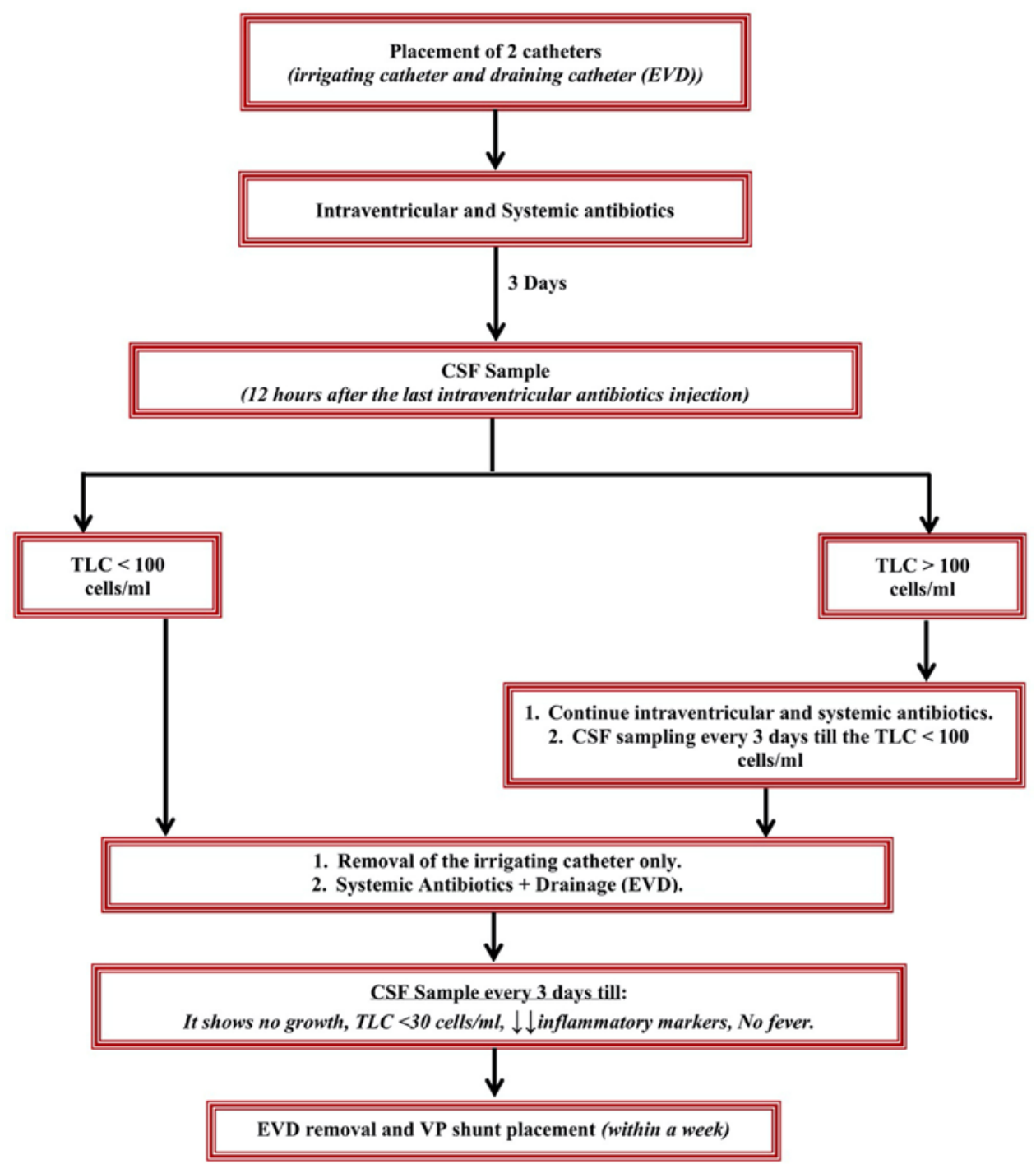

FIG. 3. Flowchart illustrating the management sequence of the ventricular irrigation subjects. TLC = total leukocyte count. Figure is available in color online only.

common complication and occurred in 7 patients ( 4 in the conventional group and 3 in the lavage group). One patient was found to have an entrapped temporal horn of the lateral right ventricle. One patient from the lavage group suffered obstruction of the bilateral foramen of Monro, which was treated by an endoscopic septostomy and a shunt revision operation. Later, the same patient also experienced intestinal obstruction, which was operated upon by a pediatric surgeon and showed no relation to the abdominal end of the VP shunt. Finally, a patient from the lavage group had severe pneumocephalus, and conservative measures using head elevation, nasal oxygen, and intravenous fluids were sufficient to cause regression of the pneumocephalus.

\section{Discussion \\ Lavage Efficacy}

Management of ventriculitis remains controversial, with no single management strategy that can provide a good outcome..$^{1,3}$ Plans for the treatment of ventriculitis remain inadequate for providing a favorable outcome and definitive treatment guidelines. Recently, ventricular irrigation or lavage has been shown to be effective and safe in treating such cases., ${ }^{7,10,12}$ Under current conventional management, deaths reach high levels (up to $70 \%$ )..$^{1-3}$ Although a mortality rate of $25 \%$ remains a high rate among the lavage group in our study, it is considered far better than the outcome under conventional management (52.9\%), even though it was not statistically significant. The mortality rate in the conventional group is high, which can be explained by the lower awareness of the parents in developing countries as well as the complexity and multiresistance of the causative organisms. A recent study by Shang et al. has also reported a similar mortality rate (26.3\%) among their subjects after treatment with ventricular irrigation that is comparable to the mortality rate in our lavage group. ${ }^{10}$ However, other studies conducted on a larger 
TABLE 1. Demographics of the treatment groups

\begin{tabular}{lcccc}
\hline \multicolumn{1}{c}{ Variable } & $\begin{array}{c}\text { Conventional } \\
\text { Group }\end{array}$ & $\begin{array}{c}\text { Lavage } \\
\text { Group }\end{array}$ & $\begin{array}{c}\mathrm{p} \\
\text { Value }\end{array}$ & Total \\
\hline Demographics & 17 & 16 & & 33 \\
\hline No. of patients & $7.81 \pm 7.71$ & $4.04 \pm 5.57$ & 0.12 & \\
\hline Mean age \pm SD, yrs & $6: 11$ & $11: 5$ & 0.055 & $17: 16$ \\
\hline M/F & $7.6 \pm 3.2$ & $5.7 \pm 3.4$ & 0.22 & \\
\hline $\begin{array}{l}\text { Mean FU duration } \pm \\
\text { SD, mos }\end{array}$ & & & & \\
\hline Clinical presentation, $\mathrm{n}$ & 15 & 16 & 0.32 & 31 \\
\hline $\begin{array}{l}\text { Fever } \\
\text { Disturbed conscious- } \\
\text { ness level }\end{array}$ & 8 & 10 & 0.37 & 18 \\
\hline Vomiting & 7 & 9 & 0.39 & 16 \\
\hline Convulsions & 6 & 5 & 0.81 & 11 \\
\hline Abdominal pain & 2 & 2 & 0.95 & 4 \\
\hline Meningeal irritation & 3 & 1 & 0.32 & 4 \\
\hline
\end{tabular}

$\mathrm{FU}=$ follow-up.

scale ( $n=41$ patients) reported mortality rates of less than $5 \% .^{13}$

Among the reported studies (Table 5), the mortality rates under treatment with ventricular lavage ranged from $0 \%$ to $26.3 \% .^{7-19}$ Although our mortality rate is higher than the mean mortality rate of the other reported studies, our cases are pediatric patients, which would contribute to weaker immune systems. The favorable outcome reached up to $68.8 \%$ in the lavage group compared to only $23.5 \%$ in the conventional group ( $<<0.05)$. This would be considered better than the outcomes reported in other studies under conventional therapy management. ${ }^{1,16,20}$ Moreover, the lavage group showed significantly shorter average hospitalization duration in comparison to the conventional group, which adds to the effectiveness of lavage treatment. Terada et al. reported 6 cases in a retrospective study comparing the treatment of ventriculitis using ventricular lavage to conventional management with external drains and antibiotics. ${ }^{12}$ Chan et al. reported a case of ventricular lavage with serial changes in the patient's head positioning, which helps in draining the pus that settles in the occipital horns of the ventricles. ${ }^{14}$ Also, Tandean et al. reported a lavage and aspiration using a single burr hole in a case of intraventricular empyema after shunt infection in an infant who failed standard management. ${ }^{10,21}$ While Shang et al. reported 19 patients diagnosed with ventriculitis and treated with ventricular lavage caused by gram-negative bacteria, ${ }^{10}$ Pandey et al. reported the outcomes in 19 patients with gram-negative multi-/extensive drug-resistant bacilli. ${ }^{9}$ In another study by Wang et al., 41 patients were diagnosed with ventriculitis from 7 different centers and managed with neuroendoscopic lavage. ${ }^{13}$

In the non-English literature, two reports concerning ventricular lavage for the treatment of ventriculitis were found. First, Wada et al. reported a case of severe posttraumatic ventriculitis treated with intraventricular lavage performed for 7 days with a favorable outcome. ${ }^{19}$ Similar-

TABLE 2. Predisposing factors, causative organisms, systemic antibiotics, and $m R S$ scores in the conventional group

\begin{tabular}{ccccc}
\hline & Predisposing & & & mRS Score \\
Patient No. & Factor/Cause & Organism & Systemic Antibiotics After C/S & (0-6) \\
\hline 1 & Shunt, PM & Pseudomonas spp. & Polymyxin E & 6 \\
\hline 2 & Shunt & MRSA & Vancomycin & 4 \\
\hline 3 & Shunt & MRSA & Vancomycin & 4 \\
\hline 4 & Shunt & Enterobacter & Imipenem, ofloxacin & 5 \\
\hline 5 & Shunt, PT & MRSA & Vancomycin & 0 \\
\hline 6 & Shunt & No growth & Vancomycin & 6 \\
\hline 7 & Shunt & CoNS & Vancomycin, amikacin & 6 \\
\hline 8 & Shunt & Klebsiella & Amikacin & 6 \\
\hline 9 & Shunt, PS & CoNS & Vancomycin, amikacin & 0 \\
\hline 10 & Shunt, PS & No growth & Vancomycin, amikacin & 6 \\
\hline 11 & Blood-borne & Klebsiella spp. & Tigecycline & 6 \\
\hline 12 & PS & Pseudomonas spp. & Vancomycin, amikacin & 6 \\
\hline 13 & PS & MRSA & Vancomycin & 6 \\
\hline 14 & Shunt & Klebsiella & Amikacin & 6 \\
\hline 15 & PS & NG & Clindamycin & 0 \\
\hline 16 & Shunt & Staphylococcus aureus, & Vancomycin, gentamycin & 0 \\
\hline 17 & Shunt & Acinetobacter & & 4 \\
\hline Conerichia coli & Polymyxin E & 4 \\
\hline
\end{tabular}

CoNS = coagulase-negative Staphylococcus, C/S = culture and sensitivity, MRSA = methicillin-resistant Staphylococcus aureus, NG = no growth, $\mathrm{PM}=$ postmeningitic, $\mathrm{PS}=$ postsurgical, $\mathrm{PT}=$ posttraumatic. 
TABLE 3. Predisposing factors, causative organisms, systemic antibiotics, and mRS scores in the lavage/irrigation group

\begin{tabular}{rcccc}
\hline $\begin{array}{r}\text { Patient } \\
\text { No. }\end{array}$ & $\begin{array}{c}\text { Predisposing } \\
\text { Factor/ Cause }\end{array}$ & Organism & Systemic Antibiotics After & mRS Score \\
\hline 1 & Shunt & CoNS & Amikacin, linezolid & 0 \\
\hline 2 & Shunt & No growth & Vancomycin & 0 \\
\hline 3 & Shunt & CoNS & Amikacin & 0 \\
\hline 4 & Shunt, PM & Klebsiella & Imipenem, polymyxin E & 0 \\
\hline 5 & Shunt & Stenotrophomonas & Ceftazidime, amikacin & 0 \\
\hline 6 & Shunt & CoNS & Vancomycin & 0 \\
\hline 7 & Shunt & Pseudomonas spp. & Meropenem, amikacin & 0 \\
\hline 8 & Shunt & No growth & Vancomycin & 2 \\
\hline 9 & Shunt & Acinetobacter, Klebsiella spp. & Polymyxin E, imipenem & 1 \\
\hline 10 & Shunt & Candida albicans & Amphotericin B, nystatin & 6 \\
\hline 11 & Shunt & E. coli, Klebsiella & Meropenem, amikacin & 5 \\
\hline 12 & PS & MRSA & Vancomycin, amikacin & 0 \\
\hline 13 & PM & Pseudomonas & Polymyxin E & 6 \\
\hline 14 & Shunt & Klebsiella & Amikacin & 6 \\
\hline 15 & Shunt & No growth & Vancomycin & 6 \\
\hline 16 & Shunt & Klebsiella & Meropenem, amikacin & 1 \\
\hline
\end{tabular}

ly, Sato et al. reported successful treatment of ventriculitis secondary to intraventricular rupture of an abscess by continuous irrigation with Ringer's lactate solution for 14 days. ${ }^{18}$

\section{Surgical Technique}

The performance of early ventricular lavage helps in removing the intraventricular debris and clearance of the CSF and aids in improving the concentration of the antibiotics reaching the infected areas. Moreover, using the endoscope will help in visualizing membranes and opening them, in addition to the ability to take biopsy specimens and insert the ventricular end under vision. ${ }^{13}$ However, the availability of the required equipment and staff differs between various centers, especially in developing countries where the burden of infection is far higher. In such situations, the simple double-tube lavage technique described by Chan et al. can be applicable whenever there is no obstruction in the ventricular system. ${ }^{14}$ The doubletube technique used by our team is also a quite safe and cheap alternative when neurosurgical endoscopes are not available after exclusion of obstruction. An important tip was highlighted by Chan et al. while performing the nonendoscopic irrigation technique, which is serial changing of the patient's head position while performing the lavage to avoid sedimentation of debris and pus in the ventricular system..$^{14}$

As noted above, we used an isotonic saline solution with vancomycin for lavage due to its availability and safety. However, other authors used lactated Ringer's solution and CSF substitutes, which are physiologically more similar and closer to the composition of normal CSF.

Some authors described the possibility of adverse effects from the ventricular lavage inducing secondary lesions and the spread of infection using the lavage tech- nique. ${ }^{2,10}$ In our series, we tried to perform the lavage at a rate of $1 \mathrm{~L}$ of normal saline and $500 \mathrm{mg}$ of vancomycin over 30 minutes. Intrathecal injection of vancomycin is generally safe despite its known side effects and lack of a clear dosing regimen..$^{22}$

\section{Causative Organism}

The literature suggests that gram-positive organisms are the most frequent causative organisms of postoperative CNS infections. ${ }^{1}$ However, in our study, a larger number of cases were infected with gram-negative organisms (15/33 patients), including patients with mixed infections with both gram-positive and gram-negative bacteria. Other studies have also emphasized the burden played by gram-negative infections..$^{10,15}$ In our series, there was a significantly better outcome in gram-negative patients treated with lavage compared to the conventional group $(\mathrm{p}<0.05)$.

\section{Timing to Implant the Shunt}

Shunt placement after ventriculitis is a challenging decision. The timing of shunt placement plays an important role in deciding the durability of this shunt and the likelihood

TABLE 4. Mortality, morbidity, and hospital stay

\begin{tabular}{lccc}
\hline \multicolumn{1}{c}{ Group } & Mortality (\%) & $\begin{array}{c}\text { Good Outcome } \\
(\%)^{*}\end{array}$ & $\begin{array}{c}\text { Mean Hospital } \\
\text { Stay } \pm \text { SD (days) }\end{array}$ \\
\hline Irrigation/lavage & $4 / 16(25)$ & $11 / 16(68.8)$ & $20.5 \pm 14.2$ \\
\hline Conventional & $9 / 17(52.9)$ & $4 / 17(23.5)$ & $39.7 \pm 16.9$ \\
\hline$p$ Value & 0.1 & $<0.05$ & $<0.05$ \\
\hline
\end{tabular}

Boldface type indicates statistical significance. ${ }^{*} \mathrm{mRS}$ score $<3$. 
TABLE 5. Comparison between the current study and previous studies regarding outcome following ventricular lavage

\begin{tabular}{|c|c|c|c|c|}
\hline Authors \& Year & $\begin{array}{c}\text { No. of } \\
\text { Patients }\end{array}$ & $\begin{array}{c}\text { Mean } \\
\text { Age (yrs) }\end{array}$ & $\mathrm{M} / \mathrm{F}$ & Mortality (\%) \\
\hline Wang et al., $2017^{13}$ & 41 & 34.6 & $22: 19$ & 2/41 (4.9) \\
\hline Sato et al., $1994^{18}$ & 1 & 37 & $1: 0$ & 0 \\
\hline Wada et al., $2000^{19}$ & 1 & 44 & 1:0 & 0 \\
\hline Tabuchi \& Kadowaki, $2015^{11}$ & 2 & 64.50 & $0: 2$ & 0 \\
\hline Terada et al., $2016^{12}$ & 6 & 49.33 & $5: 1$ & 0 \\
\hline Kumar et al., $2016^{16}$ & 7 & 23.45 & $5: 2$ & $1 / 7(14.3)$ \\
\hline Lang et al., $2018^{17}$ & 4 & 36.25 & $4: 0$ & $1 / 4(25)$ \\
\hline Chan et al., $2017^{14}$ & 1 & 48 & $1: 0$ & 0 \\
\hline Tandean et al., $2018^{21}$ & 1 & $2 \mathrm{mos}$ & $1: 0$ & 0 \\
\hline Shang et al., $2018^{10}$ & 19 & 48.47 & $14: 5$ & $5 / 19(26.3)$ \\
\hline Chen et al., $2019^{15}$ & 25 & 45.6 & $20: 5$ & $5 / 25(20)$ \\
\hline Guan et al., $2019^{8}$ & 32 & 43 & $20: 12$ & $2 / 32(6.25)$ \\
\hline Pandey et al., $2019^{9}$ & 19 & 51 & $15: 4$ & 3/19 (15.8) \\
\hline Present study & 16 & 5.98 & $11: 5$ & $4 / 16(25)$ \\
\hline Total & 175 & 41.7 & $2.2: 1$ & 23/175 (13.1) \\
\hline
\end{tabular}

of reinfection. Also, the long duration of EVD insertion is directly associated with an increased possibility of CSF infection in general, and specifically reinfection after the resolution of ventriculitis. ${ }^{5}$ Our group decided to insert the shunt within 1 week after the resolution of the infection. Other authors recommend waiting at least 2 weeks after resolution of infection because of the hypothesis that proteins remain high in the CSF even after eradication of infection, and this can lead to complications such as shunt obstruction or malfunction. ${ }^{5}$ This might explain the relatively high rate of shunt obstruction in our study (21.2\%). Conversely, delaying the VP shunt insertion carries a high risk of reinfection or superinfection, which would interfere with the primary aim of eradicating the infection. Therefore, our team finds that further evaluation and balancing the pros and cons of early versus delayed placement of the final VP shunt should be intensely studied in future research.

\section{Limitations}

Our study has limitations. Although it is the first controlled prospective study and the number of patients is larger than in the previous available controlled retrospective studies, ${ }^{12}$ the sample size is still small. The heterogeneity of the patient population is another limitation, but to our knowledge, it is the only prospective comparative study that concentrates on the pediatric age group with ventriculitis.

Another limitation is that within the lavage group 5 patients were managed with the endoscopic lavage while 11 were managed using double-tube lavage. We believe that a new study comparing the two modalities of lavage would be also valuable; however, the equipment and trained staff are not always available in all institutions, especially in developing countries. In addition, the type of causative organism might play a role in the outcome. In our study, a fair comparison between the different causative organisms could not be performed due to the small number of cases and an unequal number of patients infected with the same organisms.

\section{Conclusions}

Early ventricular lavage to wash out pus and debris, together with intravenous and intraventricular antibiotics coupled with proper timing of shunt placement and removing the EVD after curing the infection, should improve the survival rate and outcome in cerebral ventriculitis. Ventricular lavage is safe in neonates and associated with shorter hospital stay. Moreover, mortality rates were more than doubled in the conventional group in comparison with the ventricular irrigation group.

\section{Acknowledgments}

We would like to deeply thank Hala Abdelrahman, Valentina Crespo, and Marc Matthes for figure creation, editing, and technical support. This project was self-funded from the Cairo University Hospitals.

\section{References}

1. Humphreys H, Jenks PJ. Surveillance and management of ventriculitis following neurosurgery. J Hosp Infect. 2015; 89(4):281-286.

2. Inamasu J, Kuramae T, Tomiyasu K, Nakatsukasa M. Fulminant ependymitis following intraventricular rupture of brain abscess. J Infect Chemother. 2011;17(4):534-537.

3. Karageorgopoulos DE, Falagas ME. Current control and treatment of multidrug-resistant Acinetobacter baumannii infections. Lancet Infect Dis. 2008;8(12):751-762.

4. Long W, Yuan J, Liu J, et al. Multidrug resistant brain abscess due to Acinetobacter baumannii ventriculitis cleared by intraventricular and intravenous tigecycline therapy: a case report and review of literature. Front Neurol. 2018;9:518.

5. Grossman R, Schmidek HH, Quiñones-Hinojosa A. Management of suppurative intracranial infections. In: Schmidek and Sweet Operative Neurosurgical Techniques. 6th ed. Elsevier; 2012:1631-1641.

6. Yamada S, Fukuhara N, Yamaguchi-Okada M, et al. Therapeutic outcomes of transsphenoidal surgery in pediatric patients with craniopharyngiomas: a single-center study. $J$ Neurosurg Pediatr. 2018;21(6):549-562.

7. Gaderer C, Schaumann A, Schulz M, Thomale UW. Neuroendoscopic lavage for the treatment of CSF infection with hydrocephalus in children. Childs Nerv Syst. 2018;34(10): 1893-1903.

8. Guan F, Peng WC, Huang H, et al. Application of neuroendoscopic surgical techniques in the assessment and treatment of cerebral ventricular infection. Neural Regen Res. 2019;14(12): 2095-2103.

9. Pandey S, Li L, Deng XY, et al. Outcome following the treatment of ventriculitis caused by multi/extensive drug resistance gram negative bacilli; Acinetobacter baumannii and Klebsiella pneumonia. Front Neurol. 2019;9:1174.

10. Shang F, Xu Y, Wang N, et al. Diagnosis and treatment of severe neurosurgical patients with pyogenic ventriculitis caused by gram-negative bacteria. Neurol Sci. 2018;39(1):79-84.

11. Tabuchi S, Kadowaki M. Neuroendoscopic surgery for ventriculitis and hydrocephalus after shunt infection and malfunction: preliminary report of a new strategy. Asian $J$ Endosc Surg. 2015;8(2):180-184.

12. Terada Y, Mineharu Y, Arakawa Y, et al. Effectiveness of neuroendoscopic ventricular irrigation for ventriculitis. Clin Neurol Neurosurg. 2016;146:147-151. 
13. Wang F, Yao XY, Zou ZR, et al. Management of pyogenic cerebral ventriculitis by neuroendoscopic surgery. World Neurosurg. 2017;98:6-13.

14. Chan AK, Birk HS, Yue JK, et al. Bilateral external ventricular drain placement and intraventricular irrigation combined with concomitant serial prone patient positioning: a novel treatment for gravity-dependent layering in bacterial ventriculitis. Cureus. 2017;9(4):e1175.

15. Chen F, Deng X, Wang Z, et al. Treatment of severe ventriculitis caused by extensively drug-resistant Acinetobacter baumannii by intraventricular lavage and administration of colistin. Infect Drug Resist. 2019;12:241-247.

16. Kumar A, Agrawal D, Sharma BS. The role of endoscopic lavage in recalcitrant multidrug-resistant gram-negative ventriculitis among neurosurgical patients. World Neurosurg. 2016;93:315-323.

17. Lang M, Habboub G, Moore NZ, et al. Neuroendoscopic evacuation of intraventricular empyema using a side-cutting aspiration device. J Clin Neurosci. 2018;47:323-327.

18. Sato M, Oikawa T, Sasaki T, Kodama N. Brain abscess ruptured into the lateral ventricle: the usefulness of treatment by intraventricular irrigation with antibiotics. A case report. Article in Japanese. No Shinkei Geka. 1994;22(7):689-693.

19. Wada T, Kuroda K, Yoshida Y, et al. A case of posttraumatic severe ventriculitis treated by intraventricular lavage. Article in Japanese. No Shinkei Geka. 2000;28(8):737-743.

20. Srihawan C, Castelblanco RL, Salazar L, et al. Clinical characteristics and predictors of adverse outcome in adult and pediatric patients with healthcare-associated ventriculitis and meningitis. Open Forum Infect Dis. 2016;3(2):ofw077.

21. Tandean S, Hendriansyah L, Djokomuljanto S, et al. Neuroendoscopic aspiration and lavage of intraventricular empyema following shunt infection in infants. Pan Afr Med J. 2018;31:15.

22. Ng K, Mabasa VH, Chow I, Ensom MHH. Systematic review of efficacy, pharmacokinetics, and administration of intra- ventricular vancomycin in adults. Neurocrit Care. 2014;20(1): $158-171$.

\section{Disclosures}

The authors report no conflict of interest concerning the materials or methods used in this study or the findings specified in this paper.

\section{Author Contributions}

Conception and design: El Refaee, Al Menabbawy, Fleck, Schroeder, Zohdi. Acquisition of data: El Refaee, Al Menabbawy, Katri. Analysis and interpretation of data: El Refaee, $\mathrm{Al}$ Menabbawy, Zohdi. Drafting the article: El Refaee, Al Menabbawy, Zohdi. Critically revising the article: El Refaee, Soliman, Elborady, Zohdi. Reviewed submitted version of manuscript: El Refaee, Al Menabbawy, Soliman, Elborady, Fleck, Schroeder, Zohdi. Approved the final version of the manuscript on behalf of all authors: El Refaee. Statistical analysis: El Refaee, Al Menabbawy, Soliman, Zohdi. Administrative/technical/ material support: Zohdi. Study supervision: Elborady, Schroeder.

\section{Supplemental Information \\ Previous Presentations}

A part of the study was presented at the European Association of Neurological Societies meeting in October 2018 in Brussels, Belgium.

\section{Correspondence}

Ehab El Refaee: University Medicine Greifswald, Germany. e.elrefaee@googlemail.com. 